\title{
Endorsement of the HerniaSurge guidelines by the International Endohernia Society
}

\author{
R. Bittner ${ }^{1} \cdot$ F. Köckerling ${ }^{1}$
}

Received: 11 July 2017 / Accepted: 20 September 2017 / Published online: 12 January 2018

c) Springer-Verlag France SAS 2017

Guidelines are supposed to close the gap between science and daily clinical work. The development and implementation of guidelines constitute an important step towards the introduction of optimal diagnostic and therapeutic concepts with the aim to improve the quality of treatment.

A huge achievement has been accomplished by the development of these comprehensive guidelines for the treatment of groin hernias by the HerniaSurge group. On behalf of the International Endohernia Society (IEHS), which consists of a network of laparo-endoscopic surgeons who have a particular interest in inguinal hernia surgery, we have taken part in this working group and the process for the development of these "International Guidelines for Groin Hernia Management".

The IEHS endorses the guidelines and the members are persuaded that these guidelines are worldwide important landmark in improving the treatment of patients having an inguinal hernia.

R. Bittner and F. Köckerling on behalf of IEHS.

\section{Compliance with ethical standards}

Conflict of interest The authors declare that they have no competing interests.
R. Bittner

bittnerfamilie@web.de

1 International Endohernia Society, Supperstr. 19,

70565 Stuttgart, Germany 